\title{
Las posibles causas del aumento de la incidencia de la tuberculosis en Chile
}

\author{
TANIA HERRERA M.*
}

\section{Possible causes of the increase in tuberculosis incidence in Chile}

Tuberculosis (TB) continues to be one of the leading causes of morbidity and mortality worldwide. In Chile, after a period of stagnation, an increase in cases has been observed in the last two years, reaching an incidence rate of 15.7 per 100,000 inhabitants in 2018. The study seeks to elucidate whether this increase is widespread or if it occurs in specific groups, also if it corresponds to epidemiological or operational changes. A descriptive study was carry on using data from official secondary sources to analyze changes in the number of cases in the last two years according to geographic areas and risk groups. In addition, case finding and diagnosis activities of TB program was analyzed. Increase in cases was observed in 6 regions of the country and foreigner people had the largest increase compared to other risk groups, although the incidence among Chilean is not affected for this fact. The importance of foreigners in the increase of cases occurs mainly in metropolitan region, while in the other regions there are other priority risk groups. Case finding and diagnosis activities may be contribute to increase of cases in some regions, but it does not have a relevant weight in general. In conclusion, the increase of tuberculosis cases is focused on some specific areas, which reinforces the importance of local analysis and the definition of differentiated strategies.

Key words: Tuberculosis; Incidence; Morbidity; Emigrants and immigrants; Chile.

\section{Resumen}

La tuberculosis continúa siendo una de las principales causas de morbimortalidad a nivel mundial. En Chile, luego de un periodo de estancamiento, se ha observado un aumento de casos en los últimos dos años, llegando a una tasa de incidencia de 15,7 casos por 100.000 habitantes en 2018. El estudio busca dilucidar si este aumento es generalizado o si se produce en grupos especificos de la población, y en qué medida corresponde a cambios epidemiológicos u operacionales del programa. Se realizó un estudio descriptivo utilizando datos de fuentes secundarias oficiales para analizar los cambios en el número de casos de tuberculosis en los últimos dos años según áreas geográficas y grupos vulnerables. Además, se analizó el comportamiento de las actividades de pesquisa y diagnóstico de la enfermedad. Se observó aumento de casos en 6 regiones del país, siendo los extranjeros los que aumentan en mayor medida respecto a los otros grupos vulnerables, aunque la incidencia de tuberculosis en este grupo no afecta la incidencia entre los chilenos. La importancia de los extranjeros en el aumento de los casos se produce fundamentalmente en la Región Metropolitana, en cambio en las otras regiones son otros los grupos vulnerables prioritarios. En cuanto a la pesquisa y los métodos diagnósticos, éste puede ser un factor que colabora al aumento de casos en algunas regiones, pero en general no tiene un peso relevante. En conclusión, el aumento de casos de tuberculosis está focalizado a algunas áreas específicas lo que refuerza la importancia de los análisis locales y la definición de estrategias diferenciadas.

Palabras clave: Tuberculosis; Incidencia; Morbilidad; Migrantes; Chile.

* Ministerio de Salud, Gobierno de Chile. 


\section{Introducción}

La tuberculosis es actualmente la primera causa de muerte debida a un agente infeccioso a nivel mundial, estimándose que provocó 1,6 millones de muertes y 10 millones de enfermos en el año 2017. Los organismos internacionales, como Naciones Unidas y la Organización Mundial de la Salud, reconocen a la tuberculosis como una epidemia global que requiere de medidas urgentes para acelerar el progreso hacia su eliminación ${ }^{1}$.

Si bien la carga de enfermedad está disminuyendo globalmente, la velocidad con que esta disminución sucede es demasiado lenta, alcanzando sólo un 2\% de reducción de la incidencia por año. Esta cifra es muy insuficiente para alcanzar las metas propuestas por la estrategia "Fin a la Tuberculosis" (EndTB) que busca, entre otras cosas, reducir en un $90 \%$ la incidencia de tuberculosis para el año $2035^{1}$.

En Chile, luego de los éxitos históricos alcanzados en la reducción de la morbimortalidad por tuberculosis en décadas previas, hacia el año 2005 se empieza a observar un estancamiento de la tasa de incidencia en torno a los 14 casos por cada 100.000 habitantes, y en los últimos dos años un aumento de la incidencia de 1,7 puntos entre 2016 y 2018 , llegando a una tasa de 15,7 por 100.000 habitantes en el último año. Estas cifras representan un aumento de 192 casos entre los años 2016 y 2017, y de 205 casos entre los años 2017 y $2018^{2}$.

Por otra parte, en los últimos años Chile ha sufrido una serie de cambios epidemiológicos y de la operacionalización del Programa de Tuberculosis que pudiesen estar contribuyendo a este aumento de las tasas de incidencia de esta enfermedad. Entre ellos destacan el aumento de flujos migratorios desde países con alta incidencia de tuberculosis (tasas sobre 100 por 100.000 habitantes) y la incorporación de nuevas tecnologías para realizar el diagnóstico, además de una mejora en la vigilancia epidemiológica debido a la implementación de un registro nacional electrónico que permite capturar los casos desde distintas fuentes de información ${ }^{3,4,5}$.

Por lo tanto, se hace necesario analizar en mayor detalle cuáles son las situaciones que están influyendo en el aumento de la tuberculosis en Chile. El presente estudio tiene por objetivo describir los principales cambios observados en el perfil epidemiológico de las personas que enfermaron de tuberculosis en los últimos dos años para determinar si el aumento de casos es un fenómeno general o localizado a algunos grupos vulnerables. Por otra parte, el estudio busca determinar si este aumento podría estar influenciado por una mejora en las actividades de los equipos de salud en cuanto a la localización de los casos y al diagnóstico de laboratorio.

\section{Materiales y Métodos}

Corresponde a un estudio descriptivo de la situación epidemiológica y operacional del Programa de Tuberculosis chileno en relación a los posibles factores que pudiesen explicar el aumento en el número de casos de tuberculosis en los dos últimos años.

En primer lugar se analizaron los casos de tuberculosis producidos a nivel país, diferenciándolos por región y por grupo vulnerable, para observar si el aumento de los casos se produjo en un grupo o área geográfica en particular. En el caso de encontrar cambios significativos en un grupo, se analizó la tendencia epidemiológica para los últimos años.

Para este estudio la tasa de incidencia fue definida acorde a los lineamientos de la Organización Mundial de la Salud, contemplando tanto los casos nunca antes tratados como las recaídas.

La fuente de los datos para esta parte del estudio correspondió al Registro Nacional del Programa de Tuberculosis, que es el sistema oficial de vigilancia epidemiológica para la tuberculosis en Chile. Para el cálculo de las tasas de incidencia se utilizó las cifras de población disponibles en el Instituto Nacional de Estadísticas (INE) y otras fuentes estadísticas nacionales relacionadas a grupos vulnerables en particular y que se especifican en los resultados.

La segunda parte del estudio corresponde al análisis de los cambios que se han producido en cuanto a las actividades de pesquisa y al uso de nuevas tecnologías y su relación con el aumento del diagnóstico de casos de tuberculosis. La fuente de datos para este análisis correspondió a los informes de las acciones bacteriológicas recolectadas por el Laboratorio Nacional de Tuberculosis del Instituto de Salud Pública, el que monitorea a todos los laboratorios de tuberculosis de Chile.

Los descriptores estadísticos usados para el análisis corresponden a frecuencias absolutas y proporciones. Este estudio solo utiliza fuentes secundarias de datos, que están disponibles en las instituciones señaladas, por lo que no se requiere la aprobación de un comité de ética. 


\section{Resultados}

En el año 2016, en Chile se reportaron 2.548 casos incidentes de tuberculosis. Esta cifra aumentó a 2.740 casos para el año 2017 y a 2.945 para el año 2018. La tasa de incidencia para estos años corresponde a 14,$0 ; 14,8$ y 15,7 respectivamente. En la Figura 1 se observa la tendencia de las tasas de incidencia de tuberculosis para los últimos 10 años.

En la Tabla 1 se observa el número de casos de tuberculosis pulmonar diagnosticada por baciloscopía, tuberculosis pulmonar con bacteriología positiva (que además de la baciloscopía incluye cultivo, Xpert MTB/RIF y los casos en que no se especifica el tipo de confirmación bacteriológica) y la tuberculosis extra pulmonar, entre los años 2016 y 2018.

Se observa que se ha producido un aumento progresivo de casos en los tres grupos analizados, aportando un número similar de casos al aumento total del período. El mayor aumento relativo se produjo en la tuberculosis extra pulmonar, con un $26 \%$ de aumento entre loa años 2016 y 2018. El diagnóstico de tuberculosis pulmonar mediante la baciloscopía aumentó un

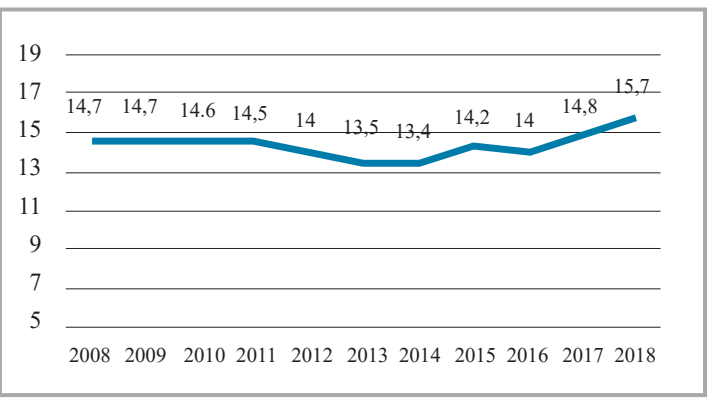

Figura 1. Tasa de incidencia de tuberculosis todas las formas. Chile 2008 -2018. Fuente: Registro Nacional de Tuberculosis/ INE.

Tabla 1. Casos de tuberculosis diferenciados por localización y método diagnóstico. Chile 2016 a 2018*

\begin{tabular}{|lccc|}
\hline & $\mathbf{2 0 1 6}$ & $\mathbf{2 0 1 7}$ & $\mathbf{2 0 1 8}$ \\
$\begin{array}{l}\text { Tuberculosis } \\
\text { pulmonar } \\
\text { baciloscopía (+) }\end{array}$ & 1.282 & 1.396 & 1.441 \\
$\begin{array}{l}\text { Tuberculosis } \\
\text { pulmonar } \\
\text { bacteriología (+) }\end{array}$ & 1.794 & 2026 & 2.132 \\
$\begin{array}{l}\text { Tuberculosis } \\
\text { extra pulmonar }\end{array}$ & 495 & 523 & 624 \\
\hline
\end{tabular}

*Fuente: Registro Nacional de Tuberculosis.
$12,4 \%$ entre estos años, y la confirmación bacteriológica por cualquiera de los métodos aumentó $18,8 \%$. Al calcular el aumento que se produce en relación al diagnóstico de tuberculosis pulmonar con confirmación bacteriológica por otros métodos distintos a la baciloscopía, el incremento es de un $34,9 \%$.

La Figura 2 muestra los casos de tuberculosis por región para los 3 años en estudio. Existen 34 casos el 2016 cuya región de residencia no está registrada por lo que fueron excluidos de este análisis.

Se observa que las regiones que presentan un aumento de casos que sigue el mismo patrón nacional son la IV, VII, VIII y la Región Metropolitana (XIII). Las regiones II y V aumentaron sus casos entre 2016 y 2017 pero se estabilizaron el año 2018.

En cuanto a los grupos vulnerables para enfermar de tuberculosis, Chile vigila la asociación de la tuberculosis con los siguientes grupos: personas viviendo con VIH (PVVIH), extranjeros, adultos mayores de 65 años (AM), diabéticos, personas en situación de calle (SC), personas pertenecientes a pueblos indígenas (PI), personas privadas de libertad (PPL), abuso de alcohol $(\mathrm{OH})$, abuso de drogas, personal de salud (PS), personas con otra inmunosupresión y contactos de casos de tuberculosis.

En el año 2016, el 63,8\% de los casos pertenecían a al menos uno de estos grupos vulnerables. En 2017 esta cifra alcanzó el 68,5\% y en el año 2018 llegó a 73\%.

La Tabla 2 muestra el porcentaje de casos de cada uno de estos grupos vulnerables respecto al total de casos de tuberculosis para los 3 años en estudio. Cabe señalar que una misma persona puede pertenecer a más de un grupo vulnerable, por lo que los porcentajes no son sumables.

Se observa que los extranjeros corresponden al principal grupo vulnerable registrado en el año 2018, desplazando a los adultos mayores y siendo el grupo que ha subido más de 10 puntos porcentuales entre 2016 y 2018 (Tabla 2). El número absoluto de personas con tuberculosis con nacionalidad distinta a la chilena que se registraron para estos años subió de 318 el 2016 a 670 el 2018, y los principales países de origen para el último año estudiado son Haití $(33,6 \%)$, Perú $(28,2 \%)$ y Bolivia $(17,5 \%)$, los tres con tasas de incidencia de tuberculosis sobre 100 por 100.000 habitantes.

El INE estimó que el porcentaje de personas 


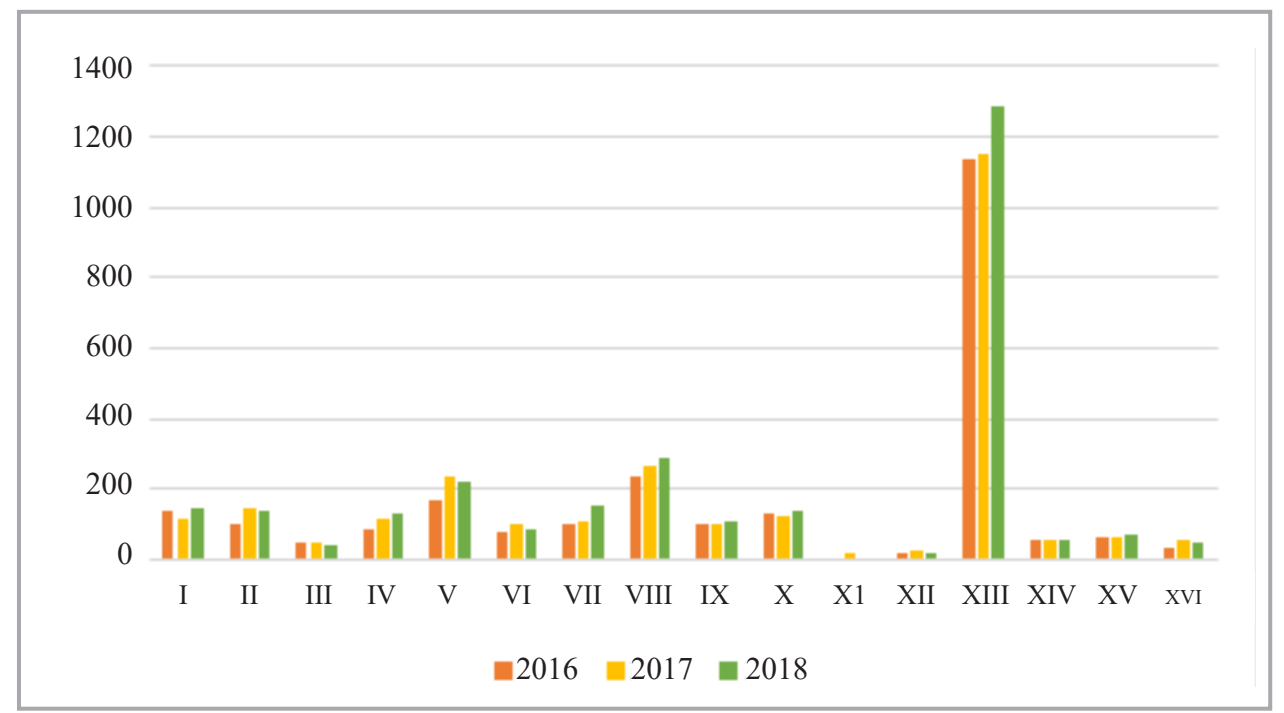

Figura 2. Casos de tuberculosis por región. Chile 2016-2018. XIII= R. Metropolitana. Fuente: Registro Nacional de Tuberculosis.

Tabla 2. Porcentaje de casos de tuberculosis que pertenecen a grupos vulnerables para tuberculosis respecto al total de casos. Chile 2016 - 2018

\begin{tabular}{|lrrr|}
\hline Grupo de riesgo & $\mathbf{2 0 1 6}$ & $\mathbf{2 0 1 7}$ & $\mathbf{2 0 1 8}$ \\
Adultos mayores & 21,6 & 21,4 & 20,3 \\
Extranjeros* & 12,5 & 15,5 & 22,8 \\
PVVIH & 8,7 & 9,6 & 8,9 \\
Diabetes & 6,9 & 7,4 & 8,2 \\
OH & 9,6 & 11,4 & 11,2 \\
Abuso drogas & 8,2 & 10,1 & 10,5 \\
PPL & 2,5 & 2,6 & 1,4 \\
PI & 4,2 & 2,5 & 4 \\
PS & 1,1 & 1,1 & 1,3 \\
SC & 2,9 & 4,7 & 4,2 \\
Contactos & 4,4 & 3,7 & 5,4 \\
Otra inmunosupresión & 3,2 & 2,9 & 4 \\
\hline
\end{tabular}

* Persona con cualquier nacionalidad distinta a la chilena, independiente de su condición migratoria. PVVIH: cohabitan con VIH; OH: alcohólicos; PPL: privados de libertad; PI: pueblos indígenas; PS: personal de salud; SC: en situación de calle. (Fuente: informes de situación epidemiológica y operacional del Programa Nacional de Control y Eliminación de la Tuberculosis 2016, 2017 y 2018).

extranjeras viviendo en Chile según el último censo de población fue un $4,4 \%$ de la población total, pero debido al flujo migratorio que se ha producido en Chile la cantidad de extranjeros corregida al 31 de diciembre de 2018 ascendería a 1.251.225 personas. Usando estas estimaciones poblacionales, la Figura 3 muestra las tasas de incidencia de tuberculosis para los últimos

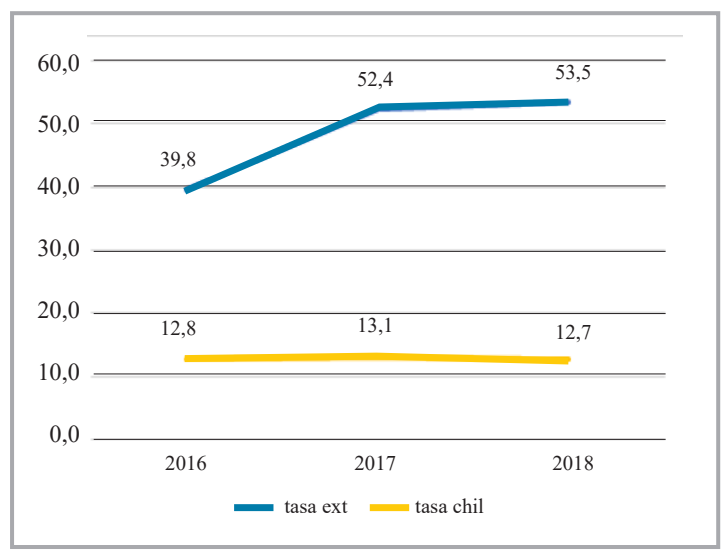

Figura 3. Incidencia de tuberculosis en chilenos (chil) y extranjeros (ext). Chile 2016 - 2018 (Fuente: Registro Nacional de Tuberculosis/ INE).

3 años diferenciándola entre chilenos y extranjeros.

Al separar los grupos se observa un aumento de la tasa de incidencia en los extranjeros en los años analizados, sin que esto tenga una repercusión en las tasas de los nacionales.

En la Tabla 3 se muestra la frecuencia relativa de los casos de tuberculosis producidos en extranjeros en las regiones que han presentado aumento de los casos en los últimos años. Se observa que si bien en todas las regiones es un grupo que ha aumentado, esta situación es especialmente significativa en la Región Metropolitana y Antofagasta, seguida por Valparaíso y la Región del Maule. En la Región del Biobío este grupo de riesgo no aparece como relevante.

La proporción de extranjeros respecto al total 
Tabla 3. Porcentajes de casos de tuberculosis en extranjeros respecto al total de casos en regiones seleccionadas. Chile 2016 -2018

\begin{tabular}{|lrrr|}
\hline Región & 2016 & 2017 & 2018 \\
Coquimbo (IV) & 3,3 & 5,3 & 8,9 \\
Del Maule (VII) & 0 & 4,5 & 12,2 \\
Biobío (VIII) & 1,5 & 1,2 & 3,5 \\
Metropolitana (XIII) & 18 & 25,3 & 32 \\
Antofagasta (II) & 29,3 & 23,4 & 36,7 \\
Valparaíso (V) & 7,6 & 8,4 & 13,1 \\
\hline
\end{tabular}

Fuente: informes de situación epidemiológica y operacional del Programa Nacional de Control y Eliminación de la Tuberculosis 2016, 2017 y 2018.

de casos no necesariamente explica el aumento de casos en la región, ya que puede suceder que se diagnostiquen más casos en extranjeros y menos casos en población nacional, lo que si bien aumenta la proporción de los primeros no produce un alza en el número de casos totales. En la Figura 4 se analizan estas mismas regiones según los cambios producidos en el número de casos, tanto nacionales como extranjeros, entre 2016 y 2018.

Se observa que en las regiones de Coquimbo, Valparaíso, del Maule y Biobío, existe un aumento mayor de los casos en personas de nacionalidad chilena respecto de los extranjeros. En Antofagasta la variación es similar para ambos grupos, en cambio en la Región Metropolitana los casos nacionales disminuyen y existe un gran aumento de casos producidos en extranjeros.

La Región del Maule explica el 15\% del aumento de casos a nivel nacional. El 32,7\% de su aumento de casos se produce en el grupo de extranjeros, ya que el año 2016 no se presentaron casos en este grupo vulnerable. La drogadicción es el segundo factor que aparece como importante en esta región, pero el mayor aumento de casos se produce en personas sin factores de riesgo.

En la Región del Biobío, entre 2016 y 2018 se produjo un aumento de 52 casos (13\% del aumento nacional), siendo la provincia de Concepción la que más aumentó (26 casos más), seguida de Arauco (22 casos más). La provincia del Biobío sube 12 casos y Talcahuano baja el número de casos en el mismo período. Cabe destacar que Arauco presenta un aumento de $100 \%$ en cuanto a los casos reportados. Para esta región, el alcoholismo y la drogadicción son los principales factores de riesgo registrados que podrían explicar el aumento en el número de casos.

La Región de Valparaíso da cuenta del 12,6\% del aumento de casos a nivel nacional. El aumento se produce fundamentalmente en el Servicio de Salud de Valparaíso - San Antonio, ya que Aconcagua aumenta solo en 2 casos y Viña del Mar - Quillota se mantiene con el mismo número de casos en 2018 respecto a 2016. En Valparaíso el grupo que más sube en número de casos corresponde al de los contactos, seguido del alcoholismo y las personas privadas de libertad.

La Región de Coquimbo explica el 11,3\% del

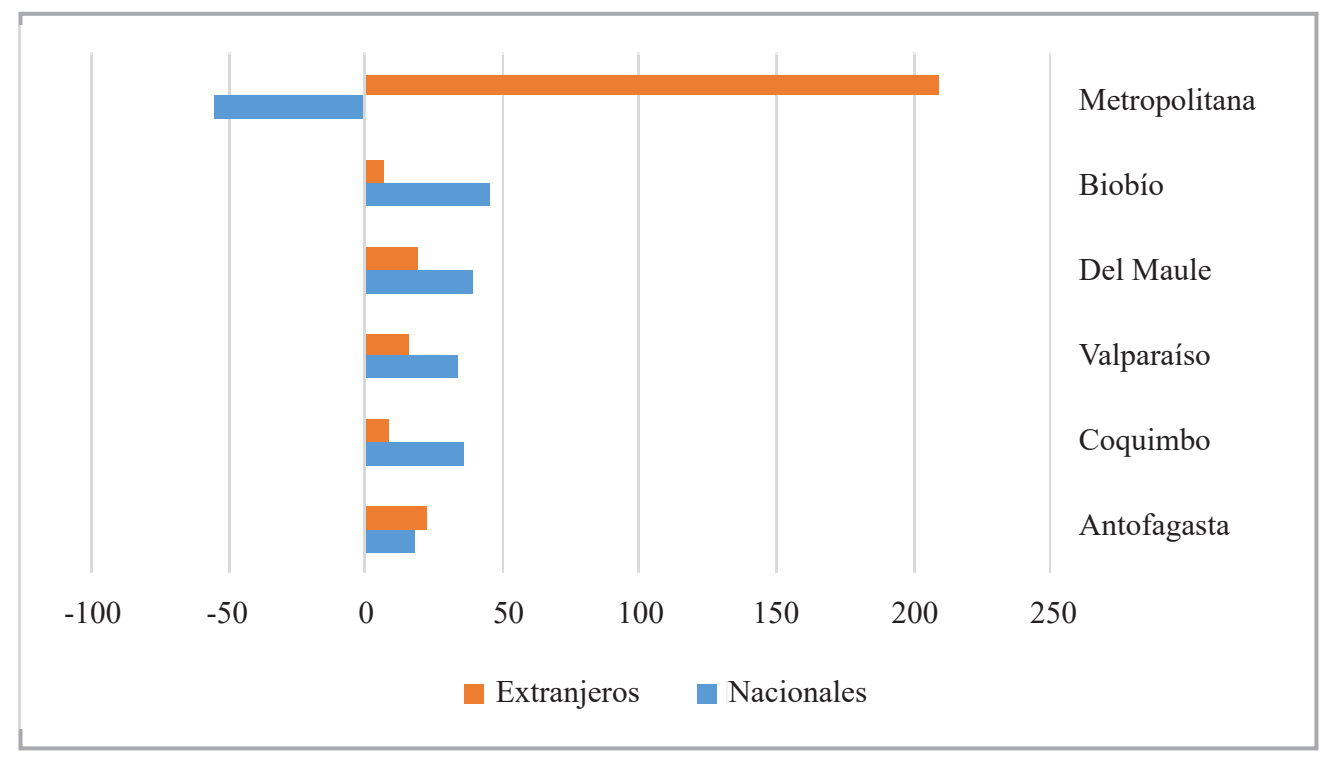

Figura 4. Variación en el número de casos de tuberculosis en nacionales y extranjeros en regiones seleccionadas. 2016 y 2018. (Fuente: Registro Nacional de Tuberculosis). 
aumento de casos a nivel nacional. El principal grupo de riesgo que sufre aumentos respecto a 2016 fue el de las personas en situación de calle, seguido de los extranjeros, el alcoholismo y la drogadicción.

La Región de Antofagasta explica el 10,1\% del aumento de los casos nacionales en el período analizado. Los extranjeros explican el 55\% del aumento de los casos, seguido del grupo de los diabéticos.

En cuanto a la Región Metropolitana, el aumento de casos entre 2016 y 2018 es de 153 casos, lo que corresponde al 39\% del aumento producido a nivel país y es la única región en la que el aumento de casos se explica por los extranjeros. El aumento de casos de tuberculosis en extranjeros en la Región Metropolitana representa el $49,4 \%$ del aumento total de este grupo en país.

La tasa de incidencia de tuberculosis aumentó de 15,3 en 2016 a 17 en 2018. En la Figura 5 se observa la evolución de la tasa de incidencia de tuberculosis y el aporte de los extranjeros en la Región Metropolitana entre los años 2014 y 2018.

Se observa que así como hay un aumento sostenido de la tasa de incidencia a lo largo del quinquenio, el aporte de los extranjeros al total de casos también va en aumento. En los dos últimos años, los casos nacionales disminuyeron con respecto a los años anteriores, por lo que el aumento de la tasa está dado por los casos en extranjeros.

La Región Metropolitana está conformada por 6 Servicios de Salud que corresponden a distintas áreas geográficas y administrativas del sistema de salud y que presentan perfiles epidemiológicos y socioeconómicos diferentes. En la Figura 6 se observan las tasas de incidencia de tuberculosis en el último quinquenio según Servicio de Salud en la Región Metropolitana.

Si bien la mayoría los Servicios de Salud de la región Metropolitana muestran una tendencia al alza en la incidencia de tuberculosis en el quinquenio analizado, las columnas presentan comportamientos diferentes. El aumento de la tasa de incidencia en el quinquenio es mayor en el SSMOCC y en el SSMS. Respecto al alza de casos producida en el país en los últimos dos años, el alza mayor se registra en el SSMC (58 casos más), seguido del SSMOCC (44 casos más) y el SSMS (42 casos más).

Al analizar el aporte de las personas extranjeras al aumento de casos en estos Servicios de Salud Metropolitanos, se observa un aumento de 56 casos extranjeros en el SSMS, 39 casos en el SSMOCC, 35 casos en el SSMN y 34 casos en el SSMC. El SSMSO aumentó 13 casos en el número de extranjeros en su cohorte 2018 respecto al 2016, en cambio en el SSMO este disminuyó en 3 casos.

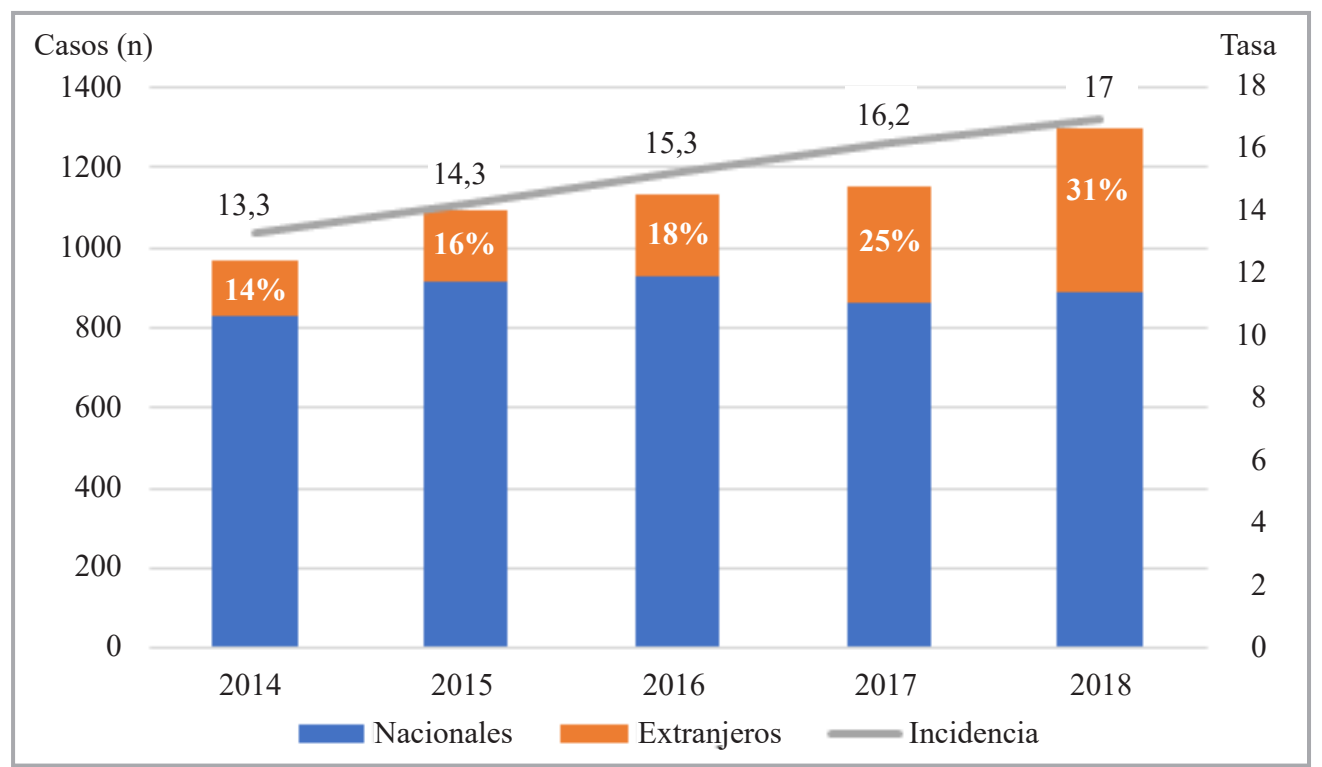

Figura 5. Tasa de incidencia y número de casos de tuberculosis en todas las formas. Región Metropolitana 2014-2018. La altura de la columna corresponde al total de casos de tuberculosis y la cifra al interior de ésta expresa el porcentaje de este total que representan los extranjeros. (Fuente: Registro Nacional de Tuberculosis). 


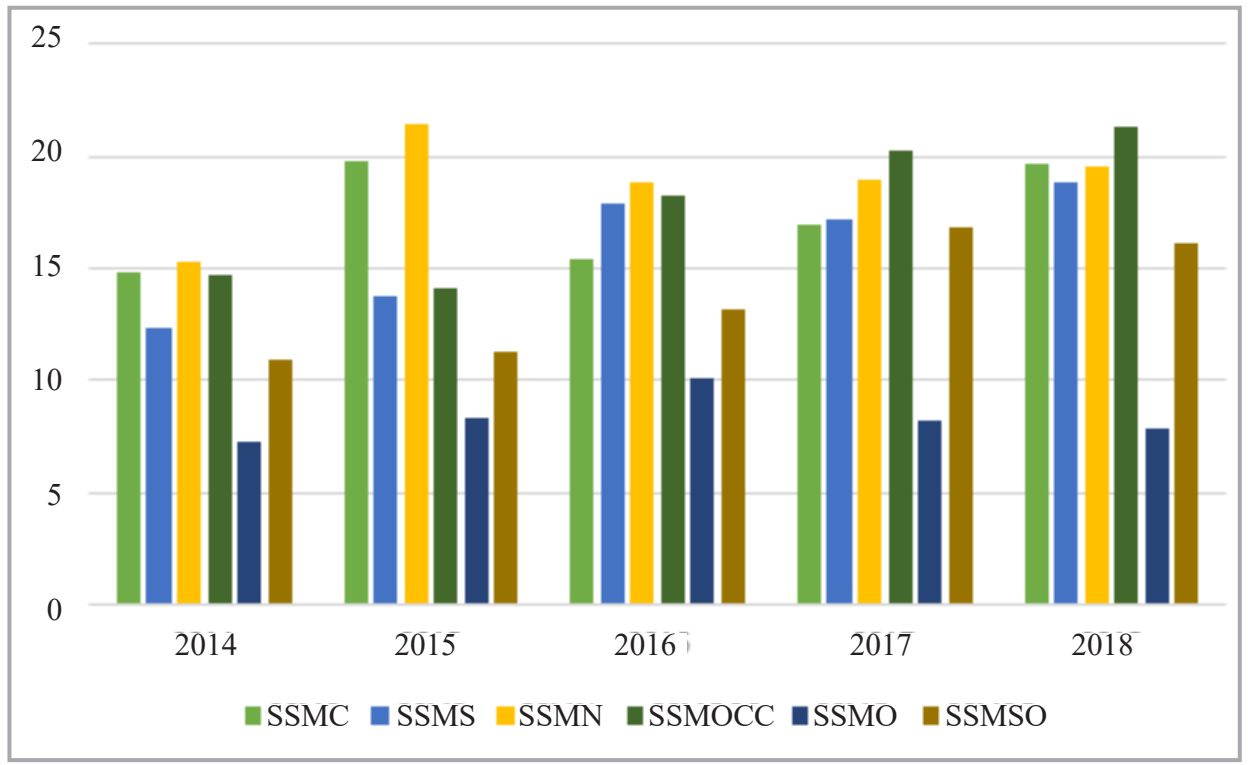

Figura 6. Incidencia de tuberculosis todas sus formas por Servicio de Salud de la Región Metropolitana $2014-2018$.

Servicio de Salud Metropolitano Central (SSMC); Servicio de Salud Metropolitano Sur (SSMS); Servicio de Salud Metropolitano Norte (SSMN); Servicio de Salud Metropolitano Occidente (SSMOCC); Servicio de Salud Metropolitano Oriente (SSMO); Servicio de Salud Metropolitano Suroriente (SSMSO). (Fuente: Registro Nacional de Tuberculosis).

Además de las variables epidemiológicas analizadas anteriormente, en la localización de los casos de tuberculosis influyen las acciones de pesquisa que se realicen por los establecimientos de salud. A grandes rasgos se esperaría que a mayor cantidad de exámenes de pesquisa, mayor sea el número de casos encontrados.

Debido a que para el año 2016 no existen datos de las acciones bacteriológicas en la región de Coquimbo, no es posible realizar el análisis incluyendo este año. Entre 2017 y 2018 número de muestras de esputo procesadas para diagnóstico de TB pulmonar, ya sea por baciloscopía o por Xpert MTB/RIF como primer examen, aumentó de 339.199 a 367.788 , es decir, se procesaron 28.589 muestras más.

Durante 2017 el número de muestras procesadas requeridas para diagnosticar un caso de tuberculosis pulmonar con confirmación bacteriológica (baciloscopía, cultivo o Xpert MTB/ RIF) fue de 167 , cifra que aumentó a 171 en 2018. Considerando el aumento en el número de muestras entre 2017 y 2018 se podría haber esperado un aumento de 171 casos en este tipo de tuberculosis; sin embargo, el aumento fue de solo 106 casos.

En la Tabla 4 se observa la variación en el número de muestras procesadas para diagnóstico de TB pulmonar de las regiones que presentaron aumento de casos en los últimos dos años, excluyendo la región de Coquimbo. Con el aumento de muestras procesadas en el período y el número de muestras que se requerían para diagnosticar un caso en el año 2016 se calculó cuántos casos deberían haberse diagnosticado (casos extra esperados 2018) y a qué porcentaje del total del aumento de casos correspondió (\% de aporte a los casos observados).

Para evaluar si el mayor número de casos de tuberculosis se produce por un diagnóstico de la enfermedad en etapas de menor carga bacilar, se consideraron dos factores: la positividad de la baciloscopía y el aporte del cultivo al diagnóstico.

En la Tabla 5 se observan estos factores para las regiones seleccionadas, excluyendo a la Región de Coquimbo.

Se deduce que las regiones se comportan heterogéneamente respecto al diagnóstico de los casos. La V y la VII región mejoran el aporte del cultivo y la positividad de la baciloscopía no aumenta, por lo que se concluye que están haciendo un diagnóstico de los casos con menores cargas bacilares. La XIII región aumenta la positividad de la baciloscopía y disminuye el aporte del cultivo, por lo que los casos se están diagnosticando en forma más tardía. La II región aumenta ambos 
Tabla 4. Muestras procesadas para diagnóstico de tuberculosis pulmonar en regiones seleccionadas.

Chile, 2016-2018

\begin{tabular}{|lrccccc|}
\hline Región & $\mathbf{2 0 1 6}$ & $\mathbf{2 0 1 8}$ & $\begin{array}{c}\text { Diferencia } \\
\mathbf{2 0 1 6 - 2 0 1 8}\end{array}$ & $\begin{array}{c}\text { Muestras } \\
\text { por caso } \\
\text { 2016 }\end{array}$ & $\begin{array}{c}\text { Casos extra } \\
\text { esperados } \\
\mathbf{2 0 1 8}\end{array}$ & $\begin{array}{c}\text { \% de aporte } \\
\text { a casos } \\
\text { observados }\end{array}$ \\
II & & & & 109 & 12 & 30 \\
V & 8.363 & 9.636 & 1.273 & 243 & 29 & 58 \\
VII & 25.509 & 32.589 & 7.083 & 263 & 1 & 1,7 \\
VIII & 17.850 & 18.218 & 368 & 269 & 11 & 21,2 \\
XIII & 44.416 & 47.306 & 2.890 & 170 & 26 & 17 \\
\hline
\end{tabular}

Fuente: Acciones bacteriológicas Laboratorio Nacional de Tuberculosis.

Tabla 5. Positividad de la baciloscopía (BK) y aporte del cultivo al diagnóstico de tuberculosis pulmonar con confirmación bacteriológica

\begin{tabular}{|lccr|r|}
\hline & \multicolumn{2}{c}{ Positividad BK } & \multicolumn{2}{c|}{ Aporte del cultivo } \\
Región & $\mathbf{2 0 1 6}$ & $\mathbf{2 0 1 8}$ & $\mathbf{2 0 1 6}$ & $\mathbf{2 0 1 8}$ \\
II & 1,2 & 1,4 & $19,4 \%$ & $26 \%$ \\
V & 0,7 & 0,7 & $16,7 \%$ & $29,7 \%$ \\
VII & 0,9 & 0,4 & $20,6 \%$ & $35 \%$ \\
VIII & 0,6 & 0,5 & $31,4 \%$ & $26 \%$ \\
XIII & 1,0 & 1,1 & $25,7 \%$ & $21,8 \%$ \\
\hline
\end{tabular}

Fuente: Acciones bacteriológicas Laboratorio Nacional de Tuberculosis. XIII: Región Metropolitana.

índices, en cambio la VIII región los disminuye, por lo que la pesquisa en etapas precoces de la enfermedad no parece ser un factor explicativo en el aumento de casos de estas regiones.

Finalmente, se describe el uso del Xpert MTB/ RIF para el diagnóstico de casos de tuberculosis pulmonar (Tabla 6). Cabe recordar que el uso a nivel programático de los equipos de Xpert MTB/RIF se produjo solo el año 2018.

De los casos de tuberculosis pulmonar con confirmación bacteriológica $8 \%$ de ellos se diagnosticaron por Xpert MTB/RIF ${ }^{13}$ durante el primer año de uso programático de esta nueva tecnología.

\section{Discusión}

Si bien Chile continúa siendo un país con una incidencia de tuberculosis relativamente baja en comparación con otros países latinoamericanos, la tendencia en los últimos años no ha sido a la baja. Según muestra la curva epidemiológica histórica, la menor tasa se produjo el $2013(13,4$ casos por 100.000 habitantes) y a partir del 2014 la tendencia ha sido al alza ${ }^{6}$. Esto se hace especialmente relevante en los últimos dos años, cuando se produjo un aumento de casi 200 casos por año entre 2016 y 2018.
El aumento de los casos de tuberculosis desde 2016 se produjo tanto en el grupo de tuberculosis pulmonar confirmada mediante bacteriología, que corresponden a los casos epidemiológicamente importantes al ser los casos contagiosos, pero también en los casos de tuberculosis extra pulmonar. La variación es proporcionalmente más importante en los casos de tuberculosis confirmados por otros métodos diagnósticos bacteriológicos distintos a la baciloscopía, lo que va en línea con el hecho de que la baciloscopía es un examen menos sensible para el diagnóstico. Sin embargo, la baciloscopía fue el método que más aportó en cuanto al número de casos diagnosticados por sobre lo estimado para este período y destaca también una baja de los casos de tuberculosis pulmonar diagnosticados por clínica o sin confirmación bacteriológica.

El aumento de la tuberculosis no es homogéneo en Chile. En los años estudiados el aumento de casos se produce básicamente en 6 de las 16 regiones, en cambio las 10 regiones restantes mantienen cifras estables, a la baja, o bien no aportan gran cantidad de casos al total nacional. El estudio de Fica y cols analizó las tendencias de la incidencia de tuberculosis por región entre los años 2014 y 2017 encontrando un aumento de la morbilidad en 9 de 15 regiones $^{7}$ (hasta el 
Tabla 6. Casos de tuberculosis diagnosticados por Xpert MTB/RIF. Chile y regiones seleccionadas. 2016-2018

\begin{tabular}{|c|c|c|c|c|c|c|}
\hline \multirow[t]{2}{*}{ Región } & \multicolumn{2}{|c|}{2016} & \multicolumn{2}{|c|}{2017} & \multicolumn{2}{|c|}{2018} \\
\hline & Casos & $\%$ & Casos & $\%$ & Casos & $\%$ \\
\hline II & 0 & 0 & 0 & 0 & 11 & $10 \%$ \\
\hline IV & 0 & 0 & 0 & 0 & 0 & 0 \\
\hline V & 0 & 0 & 1 & $0,5 \%$ & 7 & $3,8 \%$ \\
\hline VII & 0 & 0 & 0 & 0 & 4 & $4,8 \%$ \\
\hline VIII & 0 & 0 & 0 & 0 & 7 & $2,8 \%$ \\
\hline XIII & 12 & $1,5 \%$ & 58 & $5,9 \%$ & 104 & $11 \%$ \\
\hline País & 14 & $0,8 \%$ & 61 & $3,8 \%$ & 165 & $8 \%$ \\
\hline
\end{tabular}

Fuente: Acciones bacteriológicas Laboratorio Nacional de Tuberculosis. XIII: Región Metropolitana.

2017 no existía la región de Nuble, zona que fue separada de la región del Biobío desde 2018). Este estudio no evidenció aumento de las tasas en la VII y VIII región, situación que sí se produce en los últimos dos años.

Una mejor evaluación de las tendencias epidemiológicas regionales requeriría de un análisis en un número mayor de años; sin embargo, esto se aleja del objetivo del presente estudio.

En cuanto a los grupos vulnerables, el análisis realizado da cuenta de la importancia relativa del grupo de extranjeros en el aumento de casos de tuberculosis a nivel nacional, pero un análisis más focalizado advierte que este factor no contribuye de igual forma en todo el país. Este factor afecta solamente a la Región Metropolitana, y dentro de ella a 4 de los 6 Servicios de Salud, donde se observa este fenómeno. Cabe hacer notar que en los últimos años, el flujo migratorio se ha desconcentrado en cuanto a su lugar de residencia, alcanzando comunas y regiones donde antes no estaban presentes, pero aún el $63,1 \%$ de los extranjeros viven en la Región Metropolitana $^{3}$.

En las otras regiones, Antofagasta presenta un mayor aumento de casos en extranjeros que en nacionales, pero en todas las demás regiones el aumento de casos en extranjeros es menor al aumento de casos entre los chilenos. Entre estas regiones destaca la situación de la Región Del Maule que en dos años cambió su perfil epidemiológico, pasando de no tener casos en extranjeros a un aporte del $12 \%$. Esto se condice con el hecho de que las comunas de Curicó y Talca son dos de las tres comunas destacadas por el INE como las de mayor aumento en la proporción de extranjeros residentes entre 2017 y $2018^{3}$.

Lo que este estudio también pone de manifiesto es la necesidad de diferenciar las tasas de incidencia de tuberculosis entre nacionales y extranjeros para tener una visión más acabada de la situación epidemiológica. La incidencia actual en el grupo de extranjeros es similar a la reportada en estudios anteriores, por lo que es de toda lógica que ante aumento de los flujos migratorios aumente el número de $\operatorname{casos}^{8}$. En los países de baja incidencia de tuberculosis, la proporción de casos en inmigrantes alcanza hasta el $70 \%$, evidenciándose además una disminución progresiva de la incidencia en los casos nativos 9 . Esta diferencia en la epidemiología es relevante a la hora de definir estrategias atingentes a cada grupo, considerando además que la transmisión de la tuberculosis entre población extranjera y población nacional ha demostrado ser baja en diversos estudios internacionales ${ }^{10}$.

Además, se requiere profundizar en el análisis de situación dentro del propio grupo de extranjeros, y cuáles son los factores que hacen que sean más vulnerables a enfermar de tuberculosis. Factores como el país de origen, el estatus migratorio, las condiciones socioeconómicas a las que se enfrentan en Chile, las comorbilidades y el acceso a la atención de salud producen diferencias en la posibilidad de enfermar de tuberculosis que requieren un abordaje especial ${ }^{10}$.

Por otra parte, entre los chilenos existen otros factores de vulnerabilidad que también son relevantes en el aumento de casos producidos en otras regiones distintas de la metropolitana, como son el abuso de sustancias (alcohol y drogas), la situación de calle, los contactos de tuberculosis, las poblaciones privadas de libertad y las personas con diabetes mellitus. La gran proporción de casos de tuberculosis que pertenecen a alguno de los grupos vulnerables confirma que la tuberculosis va focalizándose cada vez más en estos grupos, y avizora la necesidad de nuevas estrate- 
gias de pesquisa mucho más focalizadas en ellos, haciéndose cargo de las diferencias regionales ${ }^{11}$.

El Programa Nacional de Tuberculosis cuenta entre sus lineamientos programáticos con la realización de trabajo focalizado en los grupos vulnerables, incluso con indicaciones de trabajo intersectorial que deben desarrollar las Secretarías Regionales Ministeriales de Salud ${ }^{12}$. Este hecho debe ser reforzado ampliando las estrategias programáticas para la localización de casos oportuna en estos grupos.

En cuanto al trabajo realizado por los equipos de salud en la localización de casos, se observa que este factor solo es importante en la región de Valparaíso, la que además muestra una mejoría en cuanto al diagnóstico de casos con menor carga bacilar. La Región del Maule no muestra un aumento en las actividades de pesquisa, pero sí una mejora en el diagnóstico de los casos con cargas bacilares más bajas, aunque esta situación requiere de un análisis más detallado de la situación de los laboratorios de tuberculosis y de las actividades de focalización que se están realizando en la región.

En la Región Metropolitana, en cambio, las actividades de localización de casos mejoraron en cuanto al número de muestras procesadas, pero sin que este aumento explique la mayor cantidad de casos. Además, los casos se están diagnosticando en forma más tardía. La II y VIII Región, mejoraron en cuanto a la pesquisa, pero esto tampoco explica el aumento de los casos producidos, y además estas regiones presentan un comportamiento sobre la oportunidad diagnóstica sin una tendencia clara.

El tema de la pesquisa de tuberculosis, debe además analizarse agregando el factor de la calidad de la muestra. Los Servicios de Salud pueden mejorar en el número de exámenes procesados, pero si estos son de baja calidad, fundamentalmente dados por el porcentaje de saliva que se procesa entre las muestras del laboratorio, la posibilidad de diagnóstico es menor.

Finalmente, la incorporación de nuevas tecnologías aún no significa grandes cambios en el diagnóstico de la tuberculosis en Chile. A esto hay que agregar que el test Xpert MTB/RIF ${ }^{13,14}$ está reservado para ciertos grupos vulnerables como pacientes viviendo con VIH, extranjeros y otros grupos potenciales de tuberculosis con baja carga bacilar o alto riesgo de resistencia a fármacos, en los cuales el examen reemplaza a la baciloscopía, con lo que es difícil evaluar con solo el número de exámenes realizados y los casos totales aportados si realmente este examen logra mejorar la pesquisa de tuberculosis. Se requiere también de un análisis más detallado de este factor.

\section{Conclusión}

En los últimos dos años Chile presentó un aumento de casos de tuberculosis y de la tasa de incidencia de esta enfermedad mayor a lo observado en años anteriores. Este estudio analizó algunos de los factores que pudiesen haber contribuido a este fenómeno encontrando que el aumento se produce en algunas regiones específicas y que las causas son heterogéneas entre ellas. Se concluye que es necesario realizar análisis detallados en áreas cada vez más pequeñas (regionales, provinciales, comunales) para comprender la evolución de la situación epidemiológica local, ya que los análisis nacionales no permiten un diagnóstico adecuado $\mathrm{y}$, por lo tanto, tampoco delinear estrategias específicas a cada realidad.

\section{Bibliografías}

1.- World Health Organization. Global tuberculosis report 2018. Geneva. World Health Organization, 2019.

2.- Programa Nacional de Control y Eliminación de la Tuberculosis. Informe de situación epidemiológica y operacional del Programa Nacional de Tuberculosis 2018. 2019. Ministerio de Salud de Chile, 2019.

3.- Instituto Nacional de Estadísticas. Estimación de población extranjera en Chile. 2019. [Consulta: 19 de noviembre de 2019]. Disponible en: https://www.ine.cl/ docs/default-source/demogr\%C3\%A1 ficas-y-vitales/ estimaciones-personas-extranjeras/minuta-estimacionpoblacion-extranjera-en-chile-resultados-regionales. pdf?sfvrsn $=223 \mathrm{~d} 5 \mathrm{fd} 24$.

4.- HERRERA T, ARIAS F. Nuevos métodos para el diagnóstico de la tuberculosis. Rev Chil Enferm Respir 2016. 32: 254-9.

5.- HERRERA T. Tuberculosis en Chile: ¿podemos retomar el camnino hacia la eliminación? Rev Chil Enferm Respir. 2014. 30: 225-9.

6.- PEÑA C, FARGA V. Avanzando en la lucha contra la tuberculosis en Chile. Rev Chil Enferm Respir 2013; 29: 219-227.

7.- FICA A, HERRERA T, AGUILERA X. El deterioro de la tuberculosis en Chile. Rev Med Chile 2019; 147: 1042-52.

8. - GARCÍA C. Tuberculosis en grupos de riesgo en la región metropolitana 2008. Rev Chil Enferm Respir. 2010. 26: 105-111.

9. - LANGHOLZ KRISTENSEN K, LILLEBAEK T, HOLM PETERSEN J, HARGREAVES S, NELLUMS L, FRIEDLAND J, et al. Tuberculosis inci- 
dence among migrants according to migrant status: a cohort study, Denmark, 1993 to 2015. Euro Surveill. 2019. 24(44). pii=1900238.Disponible en: https://doi. org/10.2807/1560-7917.ES.2019.24.44.1900238.

10.- ACUÑA M, ALARCÓN Y, ASTORGA S, CABIESES B, CORREA M, DABANCH J, et al. Hacia una comprensión integral de la relación entre migración internacional y enfermedades infecciosas. Colegio Médico de Chile, Universidad del Desarrollo, Sociedad Chilena de Infectología, 2019.

11.- HERRERA T. Grupos de riesgo para tuberculosis en Chile. Rev Chilena Infectol 2015; 32: 15-8.
12.- Programa Nacional de Control y Eliminación de la Tuberculosis. Manual de Organización y Procedimientos del Programa Nacional de Control y Eliminación de la Tuberculosis. Ministerio de Salud de Chile, 2015.

13.- VALLEJO P, RODRÍGUEZ JC, SEARLE A, FARGA V. Ensayo Xpert MTB/RIF en el diagnóstico de tuberculosis. Rev Chil Enferm Respir 2015; 31: 127-31.

14.- HERRERA T, ARIAS F, RUIZ N. Manual operativo. Implementación del GeneXpert MTB/RIF en el Programa de Tuberculosis. Programa de Control y Eliminación de la Tuberculosis. 2017. 